\title{
ALCALÓIDES APORFINÓIDES DO GÊNERO Ocotea (LAURACEAE)
}

\author{
Sandra Maria Warumby Zanin \\ Departamento de Farmácia, Universidade Federal do Paraná, Av. Prefeito Lothário Meissner, 632, 80210-170 Curitiba - PR, Brasil \\ Ana Luísa Lacava Lordello* \\ Departamento de Química, Universidade Federal do Paraná, CP 19081, 81531-990 Curitiba - PR, Brasil
}

Recebido em 25/7/05; aceito em 9/3/06; publicado na web em 30/8/06

\begin{abstract}
APORPHINE ALKALOIDS IN Ocotea SPECIES (LAURACEAE). During the last decades several aporphinoid alkaloids of the Ocotea species have been isolated. This review describes the occurrence of the fifty four aporphinoids in seventeen different species of Ocotea: thirty nine (39) aporphine sensu stricto, four (4) oxoaporphine, five (5) 6a,7-dehydroaporphine, one (1) didehydroaporphine, one (1) C-3-O-aporphine, one (1) C-4-O-aporphine, two (2) phenanthrene, one (1) proaporphine and their ${ }^{13} \mathrm{C}$ NMR spectral data.
\end{abstract}

Keywords: aporphine alkaloids; Ocotea $;{ }^{13} \mathrm{C}$ NMR spectral data.

\section{INTRODUÇÃO}

A distribuição da família Lauraceae no mundo é de aproximadamente 1900 espécies, sendo que destas, 390 são encontradas no Brasil, contribuindo assim com cerca de $20 \%$ do total dessas espécies $^{1}$. No estado do Paraná ela está entre as famílias mais representativas ${ }^{1}$.

Dentre os gêneros mais expressivos das Lauraceae brasileiras, tem-se o gênero Ocotea. Este gênero tem despertado o interesse dos fitoquímicos brasileiros devido a sua ampla distribuição no território nacional, e ao fato de suas espécies serem muito apreciadas no comércio madeireiro, como a Ocotea porosa, popular imbuia e Ocotea odorífera, popular sassafrás ${ }^{2}$. Contudo, muitas dessas espécies estão ameaçadas de extinção, pois além do desmatamento, as espécies de Ocotea não possuem constância na frutificação, fato que dificulta sua propagação ${ }^{3}$.

Além dos lignóides, os alcalóides isoquinolínicos constituem um grupo de substâncias bastante freqüente em espécies do gênero Ocotea, embora durante o levantamento realizado foi possível observar que em espécies que acumulam lignóides não foi constatada a presença de alcalóides ${ }^{4,5}$.

Dentre os alcalóides isoquinolínicos, os aporfinóides representam um grupo grande e ainda em expansão, com mais de 500 alcalóides isolados de mais de 90 gêneros de plantas e/ou sintetizados $^{6}$. Vários alcalóides aporfinóides comumente encontrados no gênero Ocotea apresentam pronunciada bioatividade, como a nantenina (bloqueador de contração muscular, translocação de $\left.\mathrm{Ca}^{2+}\right)^{7}$, derivados da nantenina (antagonista $\alpha_{1}$-adrenoreceptor) ${ }^{8}$, coclaurina $(\text { anti-HIV })^{9}$, glaucina (citotóxica) ${ }^{10}$, derivados halogenados da predicentrina (aumento da afinidade aos receptores dopaminérgicos $\left.\mathrm{D}_{1}\right)^{11}$, dicentrina (inibição da topoisomerase $\mathrm{II}^{12}$, atividade antineoplásica ${ }^{13}$ ), dicentrinona (inibição da topoisomerase I) ${ }^{14}$, inclusive vários deles tendo sido alvo de patentes ${ }^{6}$, o que tem acarretado um grande interesse nessa classe de compostos.

Os autores Guinaudeau, Leboeuf e Cavé, em seus trabalhos de revisão de 1975 a $1994^{15-19}$, focaram a atenção no acompanhamento evolutivo dos estudos sobre os alcalóides aporfinóides, que incluem os aporfínicos sensu stricto e os sensu lato ou biogeneticamente rela-

*e-mail: lordello@quimica.ufpr.br cionados. Estas revisões merecem destaque pela clareza e praticidade de uso dos dados fornecidos, especialmente em relação aos valores de deslocamentos químicos de $\mathrm{RMN}$ de ${ }^{13} \mathrm{C}$.

Contudo, esse conjunto de dados encontra-se atualmente bastante desatualizado por conta da falta de revisões mais modernas e também, em alguns casos, de revisões posteriores dos dados espectroscópicos de substâncias já identificadas.

Com base nisto, este artigo propõe-se a fazer uma revisão da ocorrência dos alcalóides aporfinóides dentro do gênero Ocotea e correlacioná-los com seus dados de $\mathrm{RMN}$ de ${ }^{13} \mathrm{C}$ descritos na literatura, na tentativa de auxiliar na identificação dessa classe de compostos.

Em publicações mais recentes nota-se uma tendência em numerar as posições tradicionalmente conhecidas por $1 a$ e $1 b$ em $11 b$ e $11 c$ (Figura 1) nos alcalóides aporfinóides, apesar de ambas estarem de acordo com as normas da IUPAC para essa classe de $\operatorname{compostos}^{20}$.

\section{ALCALÓIDES APORFÍNOIDES NO GÊNERO Ocotea}

A Tabela 1 mostra os 54 alcalóides aporfinóides ordenados em ordem crescente do grau de oxidação (número de grupos hidroxílicos, metoxílicos e/ou metilenodioxílicos) no esqueleto básico (Figura 1), encontrados no gênero Ocotea. Os alcalóides foram agrupados mantendo-se as designações propostas por Guinaudeau e colaboradores $^{15-19}$, a saber: 39 aporfínicos sensu stricto, 4 oxoaporfínicos, 5 6a,7-diidroaporfínicos, 1 dideidroaporfínico, 1 C-3-O-aporfínico, 1 C-4-O-aporfínico, 2 fenantrenos e 1 pró-aporfínico, distribuídos em 17 diferentes espécies do gênero Ocotea.

Este levantamento permitiu observar que dentre os alcalóides aporfinóides, os aporfínicos sensu stricto são os mais comumente encontrados no gênero e que a $\mathrm{RMN}$ de ${ }^{13} \mathrm{C}$ consiste na técnica espectroscópica mais utilizada na identificação dessa classe de compostos.

Em geral, a atribuição dos sinais de $\mathrm{RMN}$ de ${ }^{13} \mathrm{C}$ para os carbonos $\mathrm{sp}^{3}$ dos anéis B e C dos alcalóides aporfínicos sensu stricto está baseada não só nos seus valores de deslocamentos químicos, mas também na multiplicidade de seus sinais. A maior dificuldade consiste na atribuição dos sinais de $\mathrm{RMN}$ de ${ }^{13} \mathrm{C}$ referente aos doze carbonos aromáticos dos anéis $\mathrm{A}$ e $\mathrm{D}$, em especial, aos carbonos $\mathrm{sp}^{2}$ oxigenados, devido a diferenças nos padrões de substituição encontrados para essa classe de alcalóides. Os carbonos $\mathrm{sp}^{2}$ não substituídos absorvem na região de 100-112 $\delta$ e normalmente indicam o 

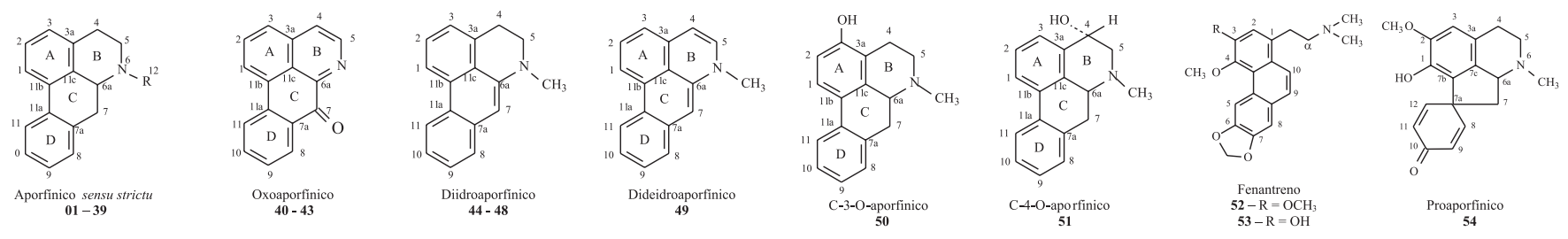

\begin{tabular}{|c|c|c|c|c|c|c|c|c|}
\hline $\begin{array}{l}\text { Posição/ } \\
\text { Alcalóide }\end{array}$ & 1 & 2 & 3 & 8 & 9 & 10 & 11 & 12 \\
\hline 1 & $\mathrm{OH}$ & $\mathrm{OCH}_{3}$ & $\mathrm{H}$ & $\mathrm{H}$ & $\mathrm{H}$ & $\mathrm{H}$ & $\mathrm{H}$ & $\mathrm{H}$ \\
\hline 2 & $\mathrm{OCH}_{3}$ & $\mathrm{OH}$ & $\mathrm{H}$ & $\mathrm{H}$ & $\mathrm{H}$ & $\mathrm{H}$ & $\mathrm{H}$ & $\mathrm{H}$ \\
\hline 3 & $\mathrm{OH}$ & $\mathrm{OCH}_{3}$ & $\mathrm{H}$ & $\mathrm{H}$ & $\mathrm{OH}$ & $\mathrm{OCH}_{3}$ & $\mathrm{H}$ & $\mathrm{H}$ \\
\hline 4 & $\mathrm{OH}$ & $\mathrm{OCH}_{3}$ & $\mathrm{H}$ & $\mathrm{H}$ & \multicolumn{2}{|c|}{$\mathrm{OCH}_{2} \mathrm{O}$} & $\mathrm{H}$ & $\mathrm{H}$ \\
\hline 5 & $\mathrm{OH}$ & $\mathrm{OCH}_{3}$ & $\mathrm{H}$ & $\mathrm{H}$ & $\mathrm{H}$ & $\mathrm{OCH}_{3}$ & $\mathrm{H}$ & $\mathrm{H}$ \\
\hline 6 & $\mathrm{OH}$ & $\mathrm{OCH}_{3}$ & $\mathrm{H}$ & $\mathrm{H}$ & $\mathrm{H}$ & $\mathrm{H}$ & $\mathrm{H}$ & $\mathrm{CH}_{3}$ \\
\hline 7 & $\mathrm{OH}$ & $\mathrm{OCH}_{3}$ & $\mathrm{H}$ & $\mathrm{H}$ & $\mathrm{H}$ & $\mathrm{OH}$ & $\mathrm{H}$ & $\mathrm{CH}_{3}$ \\
\hline 8 & $\mathrm{OH}$ & $\mathrm{OCH}_{3}$ & $\mathrm{H}$ & $\mathrm{H}$ & $\mathrm{H}$ & $\mathrm{N}\left(\mathrm{CH}_{2} \mathrm{Ph}\right)_{2}$ & $\mathrm{H}$ & $\mathrm{CH}_{3}$ \\
\hline 9 & $\mathrm{OH}$ & $\mathrm{OCH}_{3}$ & $\mathrm{H}$ & $\mathrm{H}$ & $\mathrm{OCH}_{3}$ & $\mathrm{OCH}_{3}$ & $\mathrm{H}$ & $\mathrm{CH}_{3}$ \\
\hline 10 & $\mathrm{OCH}_{3}$ & $\mathrm{OH}$ & $\mathrm{H}$ & $\mathrm{H}$ & $\mathrm{OCH}_{3}$ & $\mathrm{OCH}_{3}$ & $\mathrm{H}$ & $\mathrm{CH}_{3}^{3}$ \\
\hline 11 & $\mathrm{OCH}_{3}$ & $\mathrm{OCH}_{3}$ & $\mathrm{H}$ & $\mathrm{H}$ & $\mathrm{OCH}_{3}$ & $\mathrm{OCH}_{3}$ & $\mathrm{H}$ & $\mathrm{CH}_{3}$ \\
\hline 12 & $\mathrm{OCH}_{3}$ & $\mathrm{OCH}_{3}$ & $\mathrm{H}$ & $\mathrm{H}$ & \multicolumn{2}{|c|}{$\mathrm{OCH}_{2} \mathrm{O}$} & $\mathrm{H}$ & $\mathrm{CH}_{3}$ \\
\hline 13 & \multicolumn{2}{|c|}{$\mathrm{OCH}_{2} \mathrm{O}$} & $\mathrm{H}$ & $\mathrm{H}$ & $\mathrm{OH}$ & $\mathrm{OCH}_{3}$ & $\mathrm{H}$ & $\mathrm{CH}_{3}$ \\
\hline 14 & \multicolumn{2}{|c|}{$\mathrm{OCH}_{2}^{2} \mathrm{O}$} & $\mathrm{H}$ & $\mathrm{H}$ & $\mathrm{OCH}_{3}$ & $\mathrm{OCH}_{3}$ & $\mathrm{H}$ & $\mathrm{CH}_{3}$ \\
\hline 15 & $\mathrm{OCH}_{3}$ & $\mathrm{OCH}_{3}$ & $\mathrm{H}$ & $\mathrm{H}$ & $\mathrm{OH}^{3}$ & $\mathrm{OCH}_{3}^{3}$ & $\mathrm{H}$ & $\mathrm{CH}_{3}^{3}$ \\
\hline 16 & $\mathrm{OCH}_{3}$ & $\mathrm{OH}^{3}$ & $\mathrm{H}$ & $\mathrm{H}$ & \multicolumn{2}{|c|}{$\mathrm{OCH}_{2} \mathrm{O}$} & $\mathrm{H}$ & $\mathrm{CH}_{3}$ \\
\hline 17 & \multicolumn{2}{|c|}{$\mathrm{OCH}_{2} \mathrm{O}$} & $\mathrm{H}$ & $\mathrm{OCH}_{3}$ & & & $\mathrm{H}$ & $\mathrm{NOCH}_{3}$ \\
\hline 18 & $\mathrm{OH}$ & $\mathrm{OCH}_{3}$ & $\mathrm{H}$ & $\mathrm{H}$ & $\mathrm{H}$ & $\mathrm{OCH}_{3}$ & $\mathrm{OCH}_{3}$ & $\mathrm{CH}_{3}$ \\
\hline 19 & $\mathrm{OCH}_{3}$ & $\mathrm{OCH}_{3}$ & $\mathrm{H}$ & $\mathrm{H}$ & $\mathrm{H}$ & $\mathrm{OCH}_{3}$ & $\mathrm{OH}$ & $\mathrm{CH}_{3}$ \\
\hline 20 & $\mathrm{OCH}_{3}$ & $\mathrm{OCH}_{3}$ & $\mathrm{H}$ & $\mathrm{H}$ & $\mathrm{H}$ & $\mathrm{OCH}_{3}$ & $\mathrm{OCH}_{3}$ & $\mathrm{CH}_{3}$ \\
\hline 21 & $\mathrm{OCH}_{3}$ & $\mathrm{OCH}_{3}$ & $\mathrm{OCH}_{3}$ & $\mathrm{H}$ & $\mathrm{H}$ & $\mathrm{OCH}_{3}$ & $\mathrm{OH}$ & $\mathrm{CH}_{3}$ \\
\hline 22 & $\mathrm{OCH}_{3}^{3}$ & \multicolumn{2}{|c|}{$\mathrm{OCH}_{2} \mathrm{O}$} & $\mathrm{H}$ & $\mathrm{H}$ & $\mathrm{OCH}_{3}^{3}$ & $\mathrm{OH}$ & $\mathrm{CH}_{3}^{3}$ \\
\hline 23 & $\mathrm{OCH}_{3}$ & $\mathrm{OCH}_{3}$ & $\mathrm{OH}$ & $\mathrm{H}$ & $\mathrm{H}$ & $\mathrm{H}$ & $\mathrm{H}$ & $\mathrm{CH}_{3}$ \\
\hline 24 & $\mathrm{OCH}_{3}$ & $\mathrm{OCH}_{3}$ & $\mathrm{OCH}_{3}$ & $\mathrm{H}$ & $\mathrm{H}$ & $\mathrm{H}$ & $\mathrm{H}$ & $\mathrm{CH}_{3}$ \\
\hline 25 & $\mathrm{OH}$ & $\mathrm{OCH}_{3}$ & $\mathrm{OCH}_{3}$ & $\mathrm{H}$ & $\mathrm{H}$ & $\mathrm{OCH}_{3}$ & $\mathrm{OCH}_{3}$ & $\mathrm{CH}_{3}$ \\
\hline 26 & $\mathrm{OH}$ & $\mathrm{OCH}_{3}$ & $\mathrm{H}$ & $\mathrm{H}$ & $\mathrm{H}$ & $\mathrm{OCH}_{3}$ & $\mathrm{H}$ & $\mathrm{CH}_{3}$ \\
\hline 27 & \multicolumn{2}{|c|}{$\mathrm{OCH}_{2} \mathrm{O}$} & $\mathrm{OCH}_{3}$ & $\mathrm{H}$ & $\mathrm{OCH}_{3}$ & $\mathrm{OCH}_{3}$ & $\mathrm{H}$ & $\mathrm{CH}_{3}$ \\
\hline 28 & \multicolumn{2}{|c|}{$\mathrm{OCH}_{2}^{2} \mathrm{O}$} & $\mathrm{H}$ & $\mathrm{OCH}_{3}$ & $\mathrm{OCH}_{3}$ & $\mathrm{OCH}_{3}$ & $\mathrm{H}$ & $\mathrm{CH}_{3}$ \\
\hline 29 & \multicolumn{2}{|c|}{$\mathrm{OCH}_{2}^{2} \mathrm{O}$} & $\mathrm{H}$ & $\mathrm{OH}^{3}$ & $\mathrm{OCH}_{3}^{3}$ & $\mathrm{OCH}_{3}^{3}$ & $\mathrm{H}$ & $\mathrm{CH}_{3}^{3}$ \\
\hline 30 & \multicolumn{2}{|c|}{$\mathrm{OCH}_{2}^{2} \mathrm{O}$} & $\mathrm{OCH}_{3}$ & $\mathrm{OH}$ & $\mathrm{OCH}_{3}$ & $\mathrm{OCH}_{3}$ & $\mathrm{H}$ & $\mathrm{CH}_{3}$ \\
\hline 31 & \multicolumn{2}{|c|}{$\mathrm{OCH}_{2}^{2} \mathrm{O}$} & $\mathrm{OCH}_{3}$ & $\mathrm{OCH}_{3}$ & $\mathrm{OCH}_{3}$ & $\mathrm{OCH}_{3}$ & $\mathrm{H}$ & $\mathrm{CH}_{3}^{3}$ \\
\hline 32 & \multirow{2}{*}{\multicolumn{2}{|c|}{$\begin{array}{l}\mathrm{OCH}_{2} \mathrm{O} \\
\mathrm{OCH}_{2} \mathrm{O}\end{array}$}} & $\mathrm{H}$ & $\mathrm{OCH}_{2} \mathrm{O}$ & $\mathrm{OCH}_{3}$ & $\mathrm{H}$ & $\mathrm{CH}_{3}$ & - \\
\hline 33 & & & $\mathrm{OCH}_{3}$ & $\mathrm{OCH}_{2} \mathrm{O}$ & $\mathrm{OCH}_{3}$ & $\mathrm{H}$ & $\mathrm{CH}_{3}$ & - \\
\hline 34 & $\mathrm{OCH}_{3}$ & $\mathrm{OCH}_{3}$ & $\mathrm{OH}^{3}$ & $\mathrm{H}^{2}$ & $\mathrm{OCH}_{3}^{3}$ & $\mathrm{OCH}_{3}$ & $\mathrm{H}^{3}$ & $\mathrm{CH}_{3}$ \\
\hline 35 & $\mathrm{OH}$ & $\mathrm{OCH}_{3}$ & $\mathrm{H}$ & $\mathrm{H}$ & $\mathrm{OH}$ & $\mathrm{OCH}_{3}$ & $\mathrm{H}$ & $\mathrm{CH}_{3}$ \\
\hline 36 & \multicolumn{2}{|c|}{$\mathrm{OCH}_{2} \mathrm{O}$} & $\mathrm{OCH}_{3}$ & $\mathrm{OCH}_{3}$ & $\mathrm{OCH}_{3}$ & $\mathrm{OCH}_{3}^{3}$ & $\mathrm{H}$ & $\mathrm{H}^{\circ}$ \\
\hline 37 & $\mathrm{OH}$ & $\mathrm{OCH}_{3}$ & $\mathrm{H}$ & $\mathrm{H}$ & $\mathrm{OCH}_{3}$ & $\mathrm{H}$ & $\mathrm{H}$ & $\mathrm{H}$ \\
\hline 38 & \multicolumn{2}{|c|}{$\mathrm{OCH}_{2} \mathrm{O}$} & $\mathrm{H}$ & $\mathrm{H}$ & $\mathrm{OCH}_{3}$ & $\mathrm{OCH}_{3}$ & $\mathrm{H}$ & $\mathrm{H}$ \\
\hline 39 & \multicolumn{2}{|c|}{$\mathrm{OCH}_{2}^{2} \mathrm{O}$} & $\mathrm{OCH}_{3}$ & $\mathrm{H}$ & $\mathrm{OCH}_{3}$ & $\mathrm{OCH}_{3}$ & $\mathrm{H}$ & $\mathrm{H}$ \\
\hline 40 & \multicolumn{2}{|c|}{$\mathrm{OCH}_{2}^{2} \mathrm{O}$} & $\mathrm{H}$ & $\mathrm{H}$ & $\mathrm{OCH}_{3}$ & $\mathrm{OCH}_{3}$ & $\mathrm{H}$ & - \\
\hline 41 & \multicolumn{2}{|c|}{$\mathrm{OCH}_{2} \mathrm{O}$} & $\mathrm{H}$ & $\mathrm{H}$ & $\mathrm{H}$ & $\mathrm{OCH}_{2} \mathrm{O}$ & - & - \\
\hline 42 & & & $\mathrm{OCH}_{3}$ & $\mathrm{H}$ & $\mathrm{OCH}_{3}$ & $\mathrm{OCH}_{3}$ & $\mathrm{H}$ & - \\
\hline 43 & & & $\mathrm{OCH}_{3}^{3}$ & $\mathrm{OCH}_{3}$ & $\mathrm{OCH}_{3}^{3}$ & $\mathrm{OCH}_{3}^{3}$ & $\mathrm{H}$ & - \\
\hline 44 & $\mathrm{OCH}_{3}$ & $\mathrm{OCH}_{3}$ & $\mathrm{H}$ & $\mathrm{H}$ & & & $\mathrm{H}$ & $\mathrm{CH}_{3}$ \\
\hline 45 & & & $\mathrm{H}$ & $\mathrm{H}$ & $\mathrm{OCH}_{3}$ & $\mathrm{OCH}_{3}$ & $\mathrm{H}$ & $\mathrm{CH}_{3}$ \\
\hline 46 & & & $\mathrm{OCH}_{3}$ & $\mathrm{H}$ & $\mathrm{OCH}_{3}$ & $\mathrm{OCH}_{3}$ & $\mathrm{H}$ & $\mathrm{CH}_{3}$ \\
\hline 47 & & & $\mathrm{H}$ & $\mathrm{OCH}_{3}$ & $\mathrm{OCH}_{3}$ & $\mathrm{OCH}_{3}$ & $\mathrm{H}$ & $\mathrm{CH}_{3}$ \\
\hline 48 & $\mathrm{OCH}_{3}$ & $\mathrm{OCH}_{3}$ & $\mathrm{OH}$ & $\mathrm{H}^{3}$ & $\mathrm{H}^{3}$ & $\mathrm{H}$ & $\mathrm{H}$ & $\mathrm{CH}_{3}$ \\
\hline 49 & & & $\mathrm{OCH}_{3}$ & $\mathrm{H}$ & $\mathrm{OCH}_{3}$ & $\mathrm{OCH}_{3}$ & $\mathrm{H}$ & $\mathrm{CH}_{3}$ \\
\hline 50 & & & $\mathrm{OH}^{3}$ & $\mathrm{H}$ & $\mathrm{OCH}_{3}^{3}$ & $\mathrm{OCH}_{3}$ & $\mathrm{H}$ & $\mathrm{CH}_{3}^{3}$ \\
\hline 51 & & & $\mathrm{H}$ & $\mathrm{H}$ & $\mathrm{OCH}_{3}$ & $\mathrm{OCH}_{3}$ & $\mathrm{H}$ & $\mathrm{CH}_{3}$ \\
\hline
\end{tabular}

Figura 1. Alcalóides aporfínóides identificados no gênero Ocotea 
Tabela 1. Alcalóides aporfinóides identificados em espécies do gênero Ocotea, família Lauraceae

\begin{tabular}{|c|c|c|c|c|}
\hline Alcalóide & Alcalóides & Espécie isolada & Ref. Gênero/Espécie & Ref. Carbono 13 \\
\hline \multicolumn{5}{|c|}{ Alcalóide aporfínico stricto sensu } \\
\hline Caaverina & 1 & O. glaziovii & 22 & 16 \\
\hline Asimilobina & 2 & O. glaziovii & 22 & 16 \\
\hline Laureliptina & 3 & O. caesia & 23 & 23 \\
\hline Nordomesticina & 4 & O. sinuata & 24 & - \\
\hline Zenquerina & 5 & O. caesia & 23 & 18 \\
\hline Lirinidina & 6 & O. glaziovii & 22 & 16 \\
\hline Apoglaziovina & 7 & O. variabilis & 25 & 16 \\
\hline Variabilina & 8 & O. variabilis & 25 & - \\
\hline Taliporfina & 9 & Ocotea $s p$ & 17 & $16,38,39$ \\
\hline \multirow[t]{5}{*}{ Predicentrina } & 10 & O. brachybotra & 26 & $16,30,38,39$ \\
\hline & & O. macropoda & 27 & - \\
\hline & & O. minarum & 28 & - \\
\hline & & O. vellosiana & 29 & - \\
\hline & & O. puberula & 30 & - \\
\hline \multirow[t]{2}{*}{ Glaucina } & 11 & O. macrophylla & 31 & $16,38,39$ \\
\hline & & O. vellosiana & 29 & - \\
\hline \multirow[t]{2}{*}{ Nantenina } & 12 & O. macrophylla & 31 & $16,38,39$ \\
\hline & & O. variabilis & 25 & - \\
\hline Cassiticina & 13 & O. brachybotra & 26 & - \\
\hline \multirow[t]{6}{*}{ Dicentrina } & 14 & O. macropoda & 32 & $16,30,38,39$ \\
\hline & & O. minarum & 28 & - \\
\hline & & O. leucoxylon & 14 & - \\
\hline & & O. brachybotra & 26 & - \\
\hline & & O. vellosiana & 29 & - \\
\hline & & O. puberula & 30 & - \\
\hline N-metillaurotetanina & 15 & O. puberula & 30 & 30 \\
\hline Isodomesticina & 16 & O. puberula & 30 & 30 \\
\hline $\mathrm{N}$-óxido dicentrina & 17 & O. puberula & 30 & 30 \\
\hline Coridina & 18 & O. vellosiana & 29 & 19 \\
\hline \multirow[t]{5}{*}{ Isocoridina } & 19 & Ocotea $s p$ & 32 & $16,38,39$ \\
\hline & & O. macrophylla & 31 & - \\
\hline & & O. vellosiana & 29 & - \\
\hline & & O. boldridgeiana & 19 & - \\
\hline & & O. brenesii & 33 & - \\
\hline $\mathrm{O}, \mathrm{O}$ dimetilcorituberina & 20 & O. boldridgeiana & 24 & - \\
\hline Oconovina & 21 & Ocotea sp & 32 & - \\
\hline Ococriptina & 22 & Ocotea $s p$ & 32 & - \\
\hline \multirow[t]{2}{*}{ 3-hidroxinuciferina } & 23 & O. boldridgeiana & 19 & - \\
\hline & & O. brenesii & 33 & - \\
\hline 3-methoxinuciferina & 24 & O. boldridgeiana & 19 & - \\
\hline Isooconovina & 25 & O. minarum & 28 & - \\
\hline Pulcina & 26 & O. caesia & 23 & 18 \\
\hline \multirow[t]{3}{*}{ Ocoteina } & 27 & O. puberula & $30,32,34$ & 16,30 \\
\hline & & O. minarum & 28 & - \\
\hline & & O. vellosiana & 29 & - \\
\hline \multirow[t]{4}{*}{ Ocopodina } & 28 & O. macropoda & 27,32 & - \\
\hline & & O. brachybotra & 26 & - \\
\hline & & O. minarum & 28 & - \\
\hline & & O. vellosiana & 29 & - \\
\hline \multirow[t]{3}{*}{ Leucoxina } & 29 & O. brachybotra & 26 & 16,30 \\
\hline & & O. minarum & 28 & - \\
\hline & & O. puberula & 30 & - \\
\hline Ocoxilonina & 30 & Ocotea sp & 16 & - \\
\hline \multirow{3}{*}{ Leucoxilonina } & 31 & O. minarum & 28 & - \\
\hline & & O. vellosiana & 29 & - \\
\hline & & O. leucoxylon & 14 & - \\
\hline \multirow[t]{2}{*}{ Ocominarina } & 32 & O. minarum & 28 & - \\
\hline & & O. vellosiana & 29 & - \\
\hline
\end{tabular}


Tabela 1. continuação

\begin{tabular}{|c|c|c|c|c|}
\hline Alcalóide & Alcalóides & Espécie isolada & Ref. Gênero/Espécie & Ref. Carbono 13 \\
\hline \multicolumn{5}{|c|}{ Alcalóide aporfínico stricto sensu } \\
\hline \multirow[t]{2}{*}{ Ocotominarina } & 33 & O. minarum & 28 & - \\
\hline & & O. vellosiana & 29 & - \\
\hline Talbaicalidina & 34 & O. bucherii & 18 & 41 \\
\hline Isoboldina & 35 & O. caesia & 23 & 16,38 \\
\hline Norleucoxilonina & 36 & O. minarum & 28 & - \\
\hline Nororientinina & 37 & O. caesia & 23 & 23 \\
\hline Nordicentrina & 38 & O. macropoda & 27 & 19 \\
\hline O-metilcassifilina & 39 & O. vellosiana & 29 & - \\
\hline \multicolumn{5}{|c|}{ Alcalóide oxoaporfínico } \\
\hline \multirow[t]{3}{*}{ Dicentrinona } & 40 & O. minarum & 28 & - \\
\hline & & O. leucoxylon & 14 & - \\
\hline & & O. macropoda & 27 & - \\
\hline Hernandonina & 41 & Ocotea $s p$ & 15 & - \\
\hline \multirow[t]{2}{*}{ Talicminina } & 42 & O. puberula & 34 & - \\
\hline & & O. minarum & 28 & - \\
\hline Ocominarona & 43 & O. minarum & 28 & - \\
\hline
\end{tabular}

Alcalóide diidroaporfínico (6a, 7-diidroaporfínico)

\begin{tabular}{|c|c|c|c|c|}
\hline Deidronantenina & 44 & O. macrophylla & 31 & - \\
\hline Deidrodicentrina & 45 & O. macropoda & 27,32 & 16 \\
\hline Deidroocoteína & 46 & O. puberula & 34,35 & - \\
\hline Deidroocopodina & 47 & O. macropoda & 27 & - \\
\hline 3-hidroxi-6a,7-diidronuciferina & 48 & O. brenesii & 33 & - \\
\hline \multicolumn{5}{|l|}{ Alcalóide dideidroaporfínico } \\
\hline Dideidroocoteína & 49 & O. puberula & 34,35 & - \\
\hline \multicolumn{5}{|l|}{ Alcalóide C-3-O-aporfínico } \\
\hline 3-hidroxidicentrina & $\mathbf{5 0}$ & O.puberula & 30 & 30 \\
\hline \multicolumn{5}{|l|}{ Alcalóide C-4-O- aporfiínico } \\
\hline 4- hidroxidicentrina & 51 & O. minarum & 28 & - \\
\hline \multicolumn{5}{|l|}{ Alcalóide fenantreno } \\
\hline Talictuberina & 52 & O. insularis & 19 & 19 \\
\hline 3-O-dimetiltalictuberina & 53 & O. insularis & 19 & - \\
\hline \multicolumn{5}{|l|}{ Alcalóide proaporfínico } \\
\hline Glaziovina & 54 & $\begin{array}{l}\text { O. glaziovii } \\
\text { O. variabilis } \\
\text { O. brachybotra }\end{array}$ & $\begin{array}{l}32 \\
25 \\
26\end{array}$ & $\begin{array}{c}39,40 \\
- \\
-\end{array}$ \\
\hline
\end{tabular}

número de substituintes nos anéis $\mathrm{A}$ e $\mathrm{D}$, especialmente quando substituídos por grupos hidroxílicos, que não aparecem nos espectros de $\mathrm{RMN}$ de ${ }^{13} \mathrm{C}$. Os carbonos $\mathrm{sp}^{2}$ oxigenados absorvem normalmente acima de $140 \delta$ e são posicionados por comparação com dados da literatura ou, mais recentemente, por introspecção dos mapas de contorno heteronuclear a uma ou mais ligações.

A maior parte dos alcalóides identificados no gênero Ocotea, relacionados na Figura 1, apresentam substituintes oxigenados nas posições 1 e 2 (anel A) e 9 e 10 (anel D), porém a presença de substituintes oxigenados nas posições 3 (anel A) e/ou nas posições 8 e 11 (anel D) pode também ser observada no gênero, embora em menor proporção. As Tabelas 2 e 3 apresentam os $\delta$ de $\mathrm{RMN} \mathrm{de}{ }^{13} \mathrm{C}$ para todos os alcalóides aporfinóides relatados no gênero Ocotea e, dos 54 alcalóides aporfinóides identificados no gênero, somente $50 \%$ deles, ou seja, 27 tiveram seus dados de $\mathrm{RMN}$ de ${ }^{13} \mathrm{C}$ descritos na literatura científica.
Essa constatação é que nos motivou a compilar todos os dados de $\mathrm{RMN}$ de ${ }^{13} \mathrm{C}$ para essa classe de alcalóides em um único trabalho, de modo a facilitar a identificação estrutural dessa classe de substâncias.

Vale ressaltar ainda que durante este levantamento foi constatado o isolamento e a identificação de alguns alcalóides aporfinóides não usuais, dentre os quais podemos citar variabilina (8), ococriptina (22) e N-óxido dicentrina (17).

\section{CONCLUSÃO}

Durante o levantamento que originou esse artigo, observamos que a maior parte dos trabalhos que relatam o isolamento dessa classe de compostos são antigos e fornecem um número limitado de informações acerca dos dados espectroscópicos para essa classe de compostos. Contudo, em trabalhos recentes tem sido relatado um vasto e pronunciado elenco de bioatividades, o que tem provocado, 
Tabela 2. $\delta$ de $\mathrm{RMN}$ de ${ }^{13} \mathrm{C}$ observados para os alcalóides aporfínicos sensu stricto (1-38), 6a,7-diidroaporfínicos (45) e C-3-O-aporfínico (50)

\begin{tabular}{|c|c|c|c|c|c|c|c|c|c|}
\hline \multirow[t]{2}{*}{$\begin{array}{l}\text { Condições } \\
\text { de análise }\end{array}$} & DMSO $\delta$ & DMSO $\delta$ & $\begin{array}{l}\left(\mathrm{CD}_{3}\right)_{2} \mathrm{CO} \\
+\mathrm{CD}_{3} \mathrm{OD} \delta\end{array}$ & $\mathrm{CDCl}_{3} \delta$ & DMSO $\delta$ & DMSO $\delta$ & $\mathrm{CDCl}_{3} \delta$ & $\mathrm{CDCl}_{3} \delta$ & $\mathrm{CDCl}_{3} \delta$ \\
\hline & $15 \mathrm{MHz}$ & $15 \mathrm{MHz}$ & $75 \mathrm{MHz}$ & $15 \mathrm{MHz}$ & $15 \mathrm{MHz}$ & $15 \mathrm{MHz}$ & $15 \mathrm{MHz}$ & $15 \mathrm{MHz}$ & $15 \mathrm{MHz}$ \\
\hline $\begin{array}{l}\text { Alcalóide } \\
\text { /Carbono }\end{array}$ & 1 & 2 & 3 & 5 & 6 & 7 & 9 & 10 & 11 \\
\hline 1 & 141,6 & 143,2 & 141,84 & 141,5 & 141,6 & 141,6 & 140,7 & 142,3 & 143,9 \\
\hline 2 & 146,5 & 148,9 & 145,81 & 145,9 & 146,5 & 146,5 & 145,8 & 148,2 & 151,5 \\
\hline 3 & 110,9 & 115,7 & 109,67 & 110,1 & 110,3 & 110,2 & 108,7 & 113,5 & 110,1 \\
\hline $3 a$ & 127,3 & 126,8 & 122,87 & 123,9 & 127,4 & 127,5 & 123,9 & 129,6 & $127,0^{\mathrm{b}}$ \\
\hline 4 & 28,4 & 28,5 & 27,28 & 28,9 & 28,4 & 28,4 & 29,0 & 28,7 & 29,1 \\
\hline 5 & 42,7 & 42,6 & 42,64 & 43,2 & 52,9 & 52,8 & 53,5 & 53,3 & 53,1 \\
\hline $6 a$ & 53,2 & 53,2 & 53,90 & 53,8 & 62,1 & 62,5 & 62,7 & 62,5 & 62,3 \\
\hline 7 & 36,8 & 36,9 & 35,14 & 36,5 & 34,4 & 33,6 & 34,5 & 34,2 & 34,4 \\
\hline $7 a$ & 135,7 & 136,3 & 128,37 & $128,7^{\mathrm{a}}$ & 135,7 & 126,0 & 128,9 & 129,2 & 129,1 \\
\hline 8 & $128,1^{\mathrm{a}}$ & $127,7^{\mathrm{a}}$ & 114,75 & 128,1 & $128,1^{\mathrm{a}}$ & 127,9 & 110,9 & 110,7 & 110,6 \\
\hline 9 & $128,1^{\mathrm{a}}$ & $127,5^{\mathrm{a}}$ & 146,09 & $112,4^{b}$ & $127,5^{\mathrm{a}}$ & 115,4 & 147,6 & 148,1 & $147,7^{\mathrm{a}}$ \\
\hline 10 & $126,2^{\mathrm{a}}$ & $127,2^{\mathrm{a}}$ & 147,48 & 159,2 & $126,2^{\mathrm{a}}$ & 155,3 & 147,1 & 147,6 & $147,1^{\mathrm{a}}$ \\
\hline 11 & $125,9^{\mathrm{a}}$ & $126,5^{\mathrm{a}}$ & 113,45 & $114,1^{\mathrm{b}}$ & $126,0^{\mathrm{a}}$ & 113,2 & 112,0 & 110,0 & 111,4 \\
\hline $11 \mathrm{a}$ & 132,4 & 132,1 & 124,30 & 133,3 & 132,4 & 133,0 & 124,8 & 124,1 & 124,2 \\
\hline $11 \mathrm{~b}$ & 119,7 & 125,1 & 119,74 & 119,1 & 119,2 & 119,4 & 119,5 & 126,3 & 126,5 \\
\hline $11 \mathrm{c}$ & 123,5 & 129,3 & 125,12 & $129,1^{\mathrm{a}}$ & 123,5 & 122,9 & 127,2 & 125,9 & 128,6 \\
\hline $\mathrm{N}-\mathrm{CH}_{3}-12$ & - & - & - & - & 43,6 & 43,5 & 44,0 & 43,8 & 43,4 \\
\hline $\mathrm{OCH}_{3}-1$ & - & 59,3 & - & - & - & - & - & 60,3 & 59,8 \\
\hline $\mathrm{OCH}_{3}-2$ & 55,8 & - & 55,79 & $56,1^{\mathrm{c}}$ & 55,8 & 55,7 & $56,0^{\mathrm{a}}$ & - & $55,5^{\mathrm{c}}$ \\
\hline $\mathrm{OCH}_{3}-3$ & - & - & - & - & - & - & - & - & - \\
\hline $\mathrm{OCH}_{3}-9$ & - & - & - & - & - & - & $56,0^{\mathrm{a}}$ & $56,0^{\mathrm{a}}$ & $55,5^{\mathrm{c}}$ \\
\hline $\mathrm{OCH}_{3}-10$ & - & - & 55,91 & $55,2^{\mathrm{c}}$ & - & - & $55,9^{\mathrm{a}}$ & $55,8^{\mathrm{a}}$ & $55,7^{\mathrm{c}}$ \\
\hline $\mathrm{OCH}_{3}-11$ & - & - & - & - & - & - & - & - & - \\
\hline $\mathrm{OCH}_{2} \mathrm{O} 1,2$ & - & - & - & - & - & - & - & - & - \\
\hline $\mathrm{OCH}_{2} \mathrm{O} 9,10$ & - & - & - & - & - & - & - & - & - \\
\hline Condições & $\begin{array}{l}\mathrm{CDCl}_{3} \delta \\
15 \mathrm{MHz}\end{array}$ & $\begin{array}{l}\mathrm{CDCl}_{3} \delta \\
15 \mathrm{MHz}^{2}\end{array}$ & $\begin{array}{l}\mathrm{CDCl}_{3} \delta \\
75 \mathrm{MHz}^{-}\end{array}$ & $\begin{array}{l}\mathrm{CDCl}_{3} \delta \\
75 \mathrm{MHz}\end{array}$ & $\begin{array}{l}\mathrm{CDCl}_{3} \delta \\
75 \mathrm{MHz}\end{array}$ & $\begin{array}{l}\mathrm{CDCl}_{3} \delta \\
\text { Ref. } 19\end{array}$ & $\begin{array}{c}\mathrm{CDCl}_{3} \delta \\
15 \mathrm{MHz}^{2}\end{array}$ & $\begin{array}{l}\mathrm{CDCl}_{3} \delta \\
15 \mathrm{MHz}^{2}\end{array}$ & $\begin{array}{c}\mathrm{CDCl}_{3} \delta \\
15 \mathrm{MHz}\end{array}$ \\
\hline $\begin{array}{l}\text { Alcalóide } \\
\text { /Carbono }\end{array}$ & 12 & 14 & 15 & 16 & 17 & 18 & 19 & 26 & 27 \\
\hline 1 & 144,0 & 141,7 & 144,1 & 142,8 & 143,5 & 144,3 & 141,7 & 141,4 & 143,2 \\
\hline 2 & 151,4 & 146,6 & 151,9 & 148,9 & 148,7 & 152,3 & $150,8^{\mathrm{a}}$ & 145,7 & 134,8 \\
\hline 3 & 110,3 & 106,1 & 110,1 & 113,6 & 106,5 & 111,3 & 110,8 & 109,5 & 139,1 \\
\hline $3 a$ & $127,0^{\mathrm{b}}$ & 126,6 & 128,6 & 128,5 & 122,4 & 126,9 & $128,8^{\mathrm{b}}$ & 123,8 & 119,1 \\
\hline 4 & 29,0 & 29,2 & 28,8 & 27,3 & 28,5 & 29,1 & 29,1 & 28,8 & 23,6 \\
\hline 5 & 52,9 & 53,6 & 53,1 & 52,8 & 65,6 & 53,0 & 52,4 & 53,3 & 53,2 \\
\hline $6 a$ & 62,1 & 62,4 & 61,4 & 62,1 & 73,8 & 63,0 & 62,6 & 62,6 & 62,3 \\
\hline 7 & 34,9 & 34,3 & 33,9 & 33,8 & 27,1 & 35,7 & 35,6 & 34,0 & 34,1 \\
\hline $7 \mathrm{a}$ & 130,4 & 128,3 & 129,8 & 129,7 & 124,4 & 131,1 & $129,6^{\mathrm{b}}$ & $127,9^{\mathrm{a}}$ & 127,4 \\
\hline 8 & 107,8 & 110,5 & 111,2 & 108,6 & 110,8 & 111,8 & 118,6 & 128,2 & 111,1 \\
\hline 9 & $146,0^{\mathrm{a}}$ & 148,2 & 145,3 & 147,2 & 149,4 & 124,8 & 110,7 & $112,4^{\mathrm{b}}$ & 147,5 \\
\hline 10 & $145,9^{\mathrm{a}}$ & 147,6 & 144,9 & 146,9 & 149,1 & 149,9 & $149,0^{\mathrm{a}}$ & 158,2 & 147,5 \\
\hline 11 & 108,4 & 111,2 & 114,0 & 107,8 & 111,8 & 142,8 & 143,6 & $114,0^{\mathrm{b}}$ & 110,0 \\
\hline $11 \mathrm{a}$ & 125,1 & 123,4 & 123,7 & 125,0 & 120,1 & 128,2 & 119,8 & 133,1 & 123,5 \\
\hline $11 \mathrm{~b}$ & 126,4 & 116,6 & 126,7 & 126,1 & 117,4 & 119,7 & 125,4 & 119,3 & 110,4 \\
\hline $11 \mathrm{c}$ & $128,2^{\mathrm{b}}$ & 126,4 & 127,0 & 125,0 & 122,1 & 124,2 & $129,8^{\mathrm{b}}$ & $128,2^{\mathrm{a}}$ & 127,4 \\
\hline $\mathrm{N}-\mathrm{CH}_{3}-12$ & 43,6 & 44,0 & 43,6 & 42,3 & 47,6 & 44,0 & 43,6 & 43,8 & 41,9 \\
\hline $\mathrm{OCH}_{3}-1$ & 59,8 & - & 60,0 & 60,4 & - & - & 61,7 & - & - \\
\hline $\mathrm{OCH}_{3}-2$ & 55,4 & - & 55,7 & - & - & 62,4 & $55,5^{\mathrm{c}}$ & $56,0^{\mathrm{c}}$ & - \\
\hline $\mathrm{OCH}_{3}-3$ & - & - & - & - & - & - & - & - & 59,3 \\
\hline $\mathrm{OCH}_{3}-9$ & - & $55,9^{\mathrm{a}}$ & - & - & 56,3 & - & - & - & 56,0 \\
\hline $\mathrm{OCH}_{3}-10$ & - & $56,1^{\mathrm{a}}$ & 55,9 & - & 56,2 & 56,4 & $55,8^{\mathrm{c}}$ & $55,2^{\mathrm{c}}$ & 55,8 \\
\hline $\mathrm{OCH}_{3}-11$ & - & - & - & - & - & 56,4 & - & - & - \\
\hline $\mathrm{OCH}_{2} \mathrm{O} 1,2$ & - & 100,2 & - & - & 100,6 & - & - & - & 100,4 \\
\hline $\mathrm{OCH}_{2}^{2} \mathrm{O} 9,10$ & 100,4 & - & - & 101,1 & - & - & - & - & - \\
\hline
\end{tabular}


Tabela 2. continuação

\begin{tabular}{|c|c|c|c|c|c|c|c|}
\hline Condições & $\begin{array}{l}\text { DMSO } \delta \\
15 \mathrm{MHz}\end{array}$ & $\begin{array}{l}\mathrm{CDCl}_{3} \delta \\
15 \mathrm{MHz}\end{array}$ & DMSO $\delta$ & $\begin{array}{c}\left(\mathrm{CD}_{3}\right)_{2} \mathrm{CO} \\
20 \mathrm{MHz}\end{array}$ & $\begin{array}{l}\mathrm{CDCl}_{3} \delta \\
15 \mathrm{MHz}\end{array}$ & $\begin{array}{l}\mathrm{CDCl}_{3} \delta \\
15 \mathrm{MHz}\end{array}$ & $\begin{array}{c}\mathrm{CDCl}_{3} / \\
\mathrm{CD}_{3} \mathrm{OD} \delta \\
75 \mathrm{MHz}\end{array}$ \\
\hline $\begin{array}{l}\text { Alcalóide } \\
\text { /Carbono }\end{array}$ & 29 & 34 & 35 & 37 & 38 & 45 & 50 \\
\hline 1 & 141,6 & 148,0 & 140,6 & 141,47 & 141,6 & 142,4 & 144,90 \\
\hline 2 & 146,0 & 138,5 & 146,5 & 145,86 & 146,6 & 144,5 & 137,28 \\
\hline 3 & 106,8 & 145,5 & 109,2 & 111,81 & 107,1 & $106,2^{a}$ & 135,22 \\
\hline $3 a$ & $126,7^{a}$ & 116,3 & 126,7 & 129,55 & 126,5 & 127,4 & 114,41 \\
\hline 4 & 28,7 & 23,3 & 28,4 & 29,50 & 29,1 & 31,0 & 21,95 \\
\hline 5 & 52,9 & 52,9 & 52,9 & 43,97 & 43,1 & 50,6 & $*$ \\
\hline $6 a$ & 61,7 & 62,8 & 62,4 & 54,98 & 53,5 & 141,0 & $*$ \\
\hline 7 & 25,8 & 34,3 & 33,7 & 37,07 & 36,4 & 100,6 & 31,35 \\
\hline $7 \mathrm{a}$ & 115,6 & 128,4 & 129,1 & 137,2 & 127,9 & 129,3 & 126,20 \\
\hline 8 & 146,8 & $111,0^{\mathrm{a}}$ & 114,9 & 112,79 & 111,0 & $106,5^{\mathrm{a}}$ & 110,42 \\
\hline 9 & 135,9 & 147,4 & 145,4 & 159,21 & 148,1 & 149,2 & 148,83 \\
\hline 10 & 150,8 & 147,5 & 145,3 & 115,77 & 147,5 & 145,8 & 148,47 \\
\hline 11 & 102,4 & $111,1^{\mathrm{a}}$ & 113,6 & 128,95 & 110,5 & 108,4 & 111,98 \\
\hline $11 \mathrm{a}$ & 125,8 & 124,4 & 123,0 & 123,93 & 123,5 & $116,8^{b}$ & 123,84 \\
\hline $11 b$ & 116,0 & 118,8 & 119,7 & 116,01 & 116,2 & $117,0^{\mathrm{b}}$ & $*$ \\
\hline $11 \mathrm{c}$ & $126,4^{\mathrm{a}}$ & 131,1 & 123,5 & 127,43 & 127,0 & 118,4 & 124,00 \\
\hline $\mathrm{N}-\mathrm{CH}_{3}-12$ & 43,5 & 44,0 & 43,6 & - & - & 40,3 & $*$ \\
\hline $\mathrm{OCH}_{3}-1$ & - & $60,1^{b}$ & - & - & - & - & - \\
\hline $\mathrm{OCH}_{3}-2$ & - & $61,0^{\mathrm{b}}$ & 55,8 & 56,72 & - & - & - \\
\hline $\mathrm{OCH}_{3}-3$ & - & - & - & - & - & - & - \\
\hline $\mathrm{OCH}_{3}^{3}-9$ & 60,2 & $55,8^{c}$ & - & 55,54 & 55,8 & $56,0^{c}$ & 56,33 \\
\hline $\mathrm{OCH}_{3}-10$ & 55,6 & $56,1^{c}$ & 55,8 & - & 56,0 & $55,6^{\mathrm{c}}$ & 56,21 \\
\hline $\mathrm{OCH}_{3}-11$ & - & - & - & - & - & - & - \\
\hline $\mathrm{OCH}_{2} \mathrm{O} 1,2$ & 100,4 & - & - & - & 100,5 & 100,6 & 101,91 \\
\hline $\mathrm{OCH}_{2}^{2} \mathrm{O} 9,10$ & - & - & - & - & - & - & - \\
\hline
\end{tabular}

* - não observado; a,b,c - sinais podem estar trocados

Tabela 3. $\delta$ de $\mathrm{RMN}$ de ${ }^{13} \mathrm{C}$ observados para o alcalóide fenantreno (52) e pró-aporfínico (54)

\begin{tabular}{|c|c|c|c|}
\hline Condições & $\begin{array}{l}\mathrm{CDCl}_{3} \delta \\
\text { Ref. } 19\end{array}$ & Condições & $\begin{array}{c}\text { DMSO- } d_{6} \\
\text { Ref. } 39\end{array}$ \\
\hline $\begin{array}{l}\text { Alcalóide/ } \\
\text { Carbono }\end{array}$ & 52 & $\begin{array}{l}\text { Alcalóide/ } \\
\text { Carbono }\end{array}$ & 54 \\
\hline 1 & 129,5 & 1 & $141,5^{\mathrm{a}}$ \\
\hline 2 & 114,4 & 2 & 147,6 \\
\hline 3 & 150,4 & 3 & 110,7 \\
\hline 4 & 145,2 & $3 a$ & 124,7 \\
\hline 5 & $106,4^{\mathrm{a}}$ & 4 & 26,8 \\
\hline 6 & 147,7 & 5 & 54,6 \\
\hline 7 & 147,0 & $6 a$ & 65,2 \\
\hline 8 & $105,3^{\mathrm{a}}$ & 7 & 46,7 \\
\hline 9 & 125,2 & $7 \mathrm{a}$ & 50,5 \\
\hline 10 & 120,9 & $7 b$ & $134,8^{\mathrm{a}}$ \\
\hline 11 & 124,7 & $7 \mathrm{c}$ & 122,0 \\
\hline 12 & 121,2 & 8 & 154,7 \\
\hline 13 & 133,3 & 9 & $127,7^{\mathrm{b}}$ \\
\hline 14 & 125,8 & 10 & 185,5 \\
\hline$\alpha$ & 32,5 & 11 & $126,7^{b}$ \\
\hline$\beta$ & 60,9 & 12 & 150,8 \\
\hline $\mathrm{N}-\left(\mathrm{CH}_{3}\right)_{2}$ & 45,4 & $\mathrm{OCH}_{3}-2$ & 56,4 \\
\hline $\mathrm{OCH}_{3}-3$ & 56,6 & $\mathrm{~N}-\mathrm{CH}_{3}-6$ & 43,3 \\
\hline $\mathrm{OCH}_{3}-4$ & 59,8 & $\mathrm{OH}-1$ & - \\
\hline $\mathrm{OCH}_{2} \mathrm{O} 6,7$ & 101,1 & $\mathrm{O}-10$ & - \\
\hline
\end{tabular}

a,b - sinais podem estar trocados de certa forma, uma retomada do interesse no isolamento e na identificação dessa classe de compostos.

\section{AGRADECIMENTO}

Às agências de fomento CNPq e Fundação Araucária pelo apoio financeiro.

\section{REFERENCIAS}

1. Shepherd, G. J.; Conhecimento de Diversidade de Plantas Terrestres do Brasil, Ed. Unicamp: São Paulo, 2000, p. 19.

2. Lorenzi, H.; Árvores Brasileiras - Manual de Identificação e Cultivo de Plantas Arbóreas Nativas do Brasil, $4^{\mathrm{a}}$ ed.; Instituto Plantarum: São Paulo, 2002. vol. 1, p. 143-144.

3. Carvalho, P. E. R.; Espécies Arbóreas Brasileiras, Embrapa Informação Tecnológica: Brasília, 2003. vol. 1, p. 318.

4. Shamma, M.; Guinaudeau, H.; Tetrahedron 1984, 40, 4795.

5. Santos, R. I. Em Farmacognosia, da Planta ao Medicamento; Simões, C. M. O.; Schenkel, E. P.; Gosman, G.; Mello, J. C .P.; Mentez, L. A.; Petrovick, P. R., orgs.; Ed. Universidade/UFRGS/Ed. da UFSC: Porto Alegre/ Florianópolis, 1999, cap. 16

6. Jia, Q.; Qiu, Z.; Nissanka, A.; Farrow, T. M.; US Patent Application, Kind Code, serial $\mathrm{n}^{\circ}$ 741215/series code 09, 2000.

7. Ribeiro, R. A.; Carmo, L. G.; Vladimirova, J.; Jurkiewicz, N. H.; Jurkiewicz, A.; Eur. J. Pharmacol. 2003, 470, 37.

8. Indra, B.; Matsunaga, K.; Hoshini, O.; Suzuki, M.; Ogasawara, H.; Ohizumi, Y.; Eur. J. Pharmacol. 2002, 437, 173.

9. Kashiwada, Y.; Aoshima, A.; Ikeshiro, Y.; Chen, Y. P.; Furukawa, H.; Itoigawa, M.; Fujioka, K. M.; Cosentino, L. M.; Natschke, S. L. M.; Lee, K. H.; Bioorg. Med.Chem. 2005, 13, 443.

10. Hoet, S.; Stevigny, C.; Block, S.; Opperdoes, F.; Colson, P.; Baldeyrou, B; Lansiaux, A.; Bailly, C.; Quetin-Leclerq, J.; Planta Med. 2004, 70, 407. 
11. Asencio, M.; Guzmán, C. H.; López, J. J.; Cassels. B. K.; Protais, P.; Chagraoui, A.; Bioorg. Med. Chem. 2005, 13, 3699.

12. Woo, S. H.; Sun, N. J.; Cassady, J. M.; Snapka, R. M.; Biochem. Pharmacol. 1999, 57, 1141 .

13. Huang, R. L.; Chen, C. C.; Huang, Y. L.; Ou, J. C.; Po Hu, C.; Chen, C. C.; Chungming, C.; Planta Med. 1998, 64, 212.

14. Zhou, B. N.; Johnson, R. K.; Mattern, M. R.; Wang, X.; Hecht, S. M.; Beck, H. T.; Ortiz, A.; Kingston, D. G. J.; J. Nat. Prod. 2000, 63, 217.

15. Guinaudeau, H.; Leboeuf, M.; Cavé, A.; Lloydia 1975, 38, 275.

16. Guinaudeau, H.; Leboeuf, M.; Cavé, A.; J. Nat. Prod. 1979, 42, 325.

17. Guinaudeau, H.; Leboeuf, M.; Cavé, A.; J. Nat. Prod. 1983, 46, 761.

18. Guinaudeau, H.; Leboeuf, M.; Cavé, A.; J. Nat. Prod. 1988, 51, 389.

19. Guinaudeau, H.; Leboeuf, M.; Cavé, A.; J. Nat. Prod. 1994, 57, 1033.

20. Giles Jr, P. M.; Pure Appl. Chem. 1999, 71, 587;. Favre, H. A.; Giles Jr, P. M.; Hellwich, K. H.; McNaught, A. D.; Moss, G. P.; Powell, W. H.; Pure Appl. Chem. 2004, 76, 1283.

21. Vecchietti, V.; Casagrande, C.; Ferrari, G.; Tetrahedron 1976, 19, 1631.

22. Gilbert, B.; Gilbert, M. E. A.; De Oliveira, M. M.; Ribeiro, O.; Wenkert, E.; Wickberg, B.; Hollstein, V.; Rapoport, H.; J. Am. Chem. Soc. 1964, 86, 694.

23. Vilegas, J. H. Y.; Gottlieb, O. R.; Kaplan, M. A. C.; Gottlieb, H. E.; Phytochemistry 1989, 28, 3577.

24. Castro, O. C. Em Phytochemical Potencial of Tropical Plants, Recent Advances in Phytochemistry; Downum, K. R.; Romeo, J. T.; Stafford, H. A., eds.; Plenum Press: New York, 1993, vol. 27, p. 65.

25. Cava, M. P.; Behforouz, M.; Mitchell, M. J.; Tetrahedron 1972, 46, 4647.

26. Vecchietti, V.; Casagrande, C.; Ferrari, G.; Farmaco 1977, 32, 767.

27. Cava, M. P.; Venkateswarlu, A.; Tetrahedron 1971, 27, 2639.
28. Vecchietti, V.; Casagrande, C.; Ferrari, G.; Severini Ricca, G.; Farmaco 1979, 34, 829

29. Garcez, W. S.; Yoshida, M.; Gottlieb, O. R.; Phytochemistry 1995, 39, 815.

30. Araujo, A.J.; Dissertação de Mestrado, Universidade Federal do Paraná, Brasil, 2000.

31. Franca, N. C.; Giesbrecht, A. M.; Gottlieb, O. R.; Magalhães, A. F.; Magalhães, E. G.; Maia, J. G. S.; Phytochemistry 1975, 14, 1671.

32. Cava, M. P.; Watanabe, Y.; Bessho, K.; Mitchell, M. J.; Rocha, A. J.; Hwang, B.; Douglas, B.; Weisbach, J. A.; Tetrahedron 1968, 20, 2437.

33. Lopes, J. A.; Barillas, W.; Gomez, J. L.; Lin, F. T.; Alrehaily, A. J.; Sharaf, M. H. M.; Schiff, P. L.; Int. J. Pharm. 1996, 34, 145.

34. Baralle, F.;Schvarzberg, N.;Vernengo, M. J.; Moltrasio, G. Y.; Giacopello, D.; Phytochemistry 1973, 12, 948.

35. Baralle, F.; Schvarzberg, N.; Vernengo, M.; Comin, J.; Experientia 1972, 28,875 .

36. Botega, C.; Pagliosa, F. M.; Bolzani, V. S.; Yoshida, M.; Gottlieb, O.; Phytochemistry 1993, 32, 1331.

37. Silva, J. G.; Barbosa-Filho, J. M.; Silva, M. S.; Lacerda, C. D. G.; Cunha, E. V. L.; Biochem. Syst. Ecol. 2002, 30, 881.

38. Jackman, L. M.; Trewella, J. C.; Moniot, J. L.; Shamma, M.; Stephens, R. L.; Wenkert, E.; Leboeuf, M.; Cavé, A.; J. Nat. Prod. 1979, 42, 437.

39. Shamma, M.; Carbon 13 NMR Shift Assignments of Amines and Alkaloids, Plenum Press: New York, 1979, p. 305.

40. De Wet, H.; Heerden, F. R.; Wyk, B. E.; Biochem. Syst. Ecol. 2004, 32, 1145 .

41. Rönsch, H.; Preiss, A.; Schreibber, K.; Fernández de Córdoba, H.; Liebigs Ann. Chem. 1983, 744. 